\title{
Rapid Screening of Invasive Fungal Infections in the Hospital Setting Using the $(1,3)-\beta$-D-glucan Assay
}

\author{
Maria M. Hornberger, MD; Andrew T. Patterson, MD; Jane H. Kerford, MD; Brittany L. Lenz, MD; \\ Adnan Mir, MD, PhD; Arturo R. Dominguez, MD
}

Disseminated candidiasis and other invasive fungal infections are a substantial cause of morbidity and mortality in immunocompromised and critically ill patients. Diagnosis of disseminated candidiasis via blood culture and skin biopsy can be unreliable and may delay treatment. (1,3)- $\beta$-D-glucan (BDG) assay is a rapid, cost-effective, noninvasive diagnostic screening tool for the dermatology hospitalist to consider.

Cutis. 2020;106:33-34, 36.

\section{Practice Gap}

Invasive fungal infections are a leading cause of morbidity and mortality among neutropenic, immunocompromised, and critically ill patients. Candida species are the most common cause of fungemia, with portals of entry into the bloodstream including the gastrointestinal tract, contaminated intravascular catheters, and localized foci of infection. ${ }^{1}$ Diagnosis of invasive candidiasis remains challenging due to an absence of specific clinical signs and symptoms, varying from a mild fever that is unresponsive to antibiotics to florid sepsis. When present, clinical clues may include chorioretinitis; muscle abscesses; and skin eruptions, characteristically with Candida tropicalis. Cutaneous manifestations of disseminated Candida infections appear in only $13 \%$ of affected patients. ${ }^{1}$ The lesions typically present as 5- to $10-\mathrm{mm}$ pink dermal papules or painless pustules on an erythematous base and may be singular, localized, or diffuse in distribution. Body regions normally involved are the trunk, arms, and legs, rarely the head and neck. ${ }^{1}$ Cutaneous lesions often develop at a time when patients are febrile, are not responding to antibiotics, and are clinically deteriorating.
A 15-year-old adolescent boy with pre-B-cell acute lymphoblastic leukemia was admitted with febrile neutropenia for presumed septic shock secondary to an unknown infectious etiology. The patient was started on broad-spectrum intravenous antibiotics, and blood cultures were obtained. On the second day of hospitalization, he developed approximately 10 to 15 discrete, 3- to 6- $\mathrm{mm}$, pink to violaceous papules scattered on the chest and arms (Figure 1). Over several hours, the number of lesions increased to more than 50 with involvement of the legs (Figure 2). A punch biopsy of lesional skin from the left dorsal wrist demonstrated a circumscribed abscess of yeast

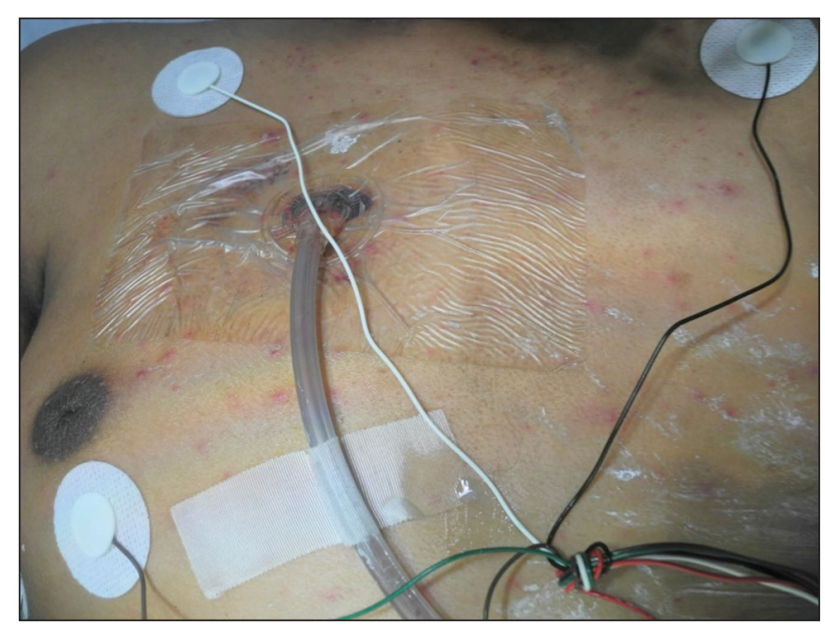

FIGURE 1. Discrete, pink to violaceous papules scattered on the chest.

Dr. Hornberger is from the Transitional Internship Program, and Drs. Patterson, Kerford, and Lenz are from the Department of Dermatology, all at the San Antonio Uniformed Services Health Education Consortium, Texas. Dr. Mir is from Dermpath Diagnostics, Port Chester, New York, and the Department of Dermatology at both Weill Cornell Medicine and New York Medical College, New York. Dr. Dominguez is from the Departments of Dermatology and Medicine, University of Texas Southwestern, Dallas.

The authors report no conflict of interest.

The views presented do not represent the official views of the Department of Defense or its components.

Correspondence: Maria M. Hornberger, MD, 3551 Roger Brooke Dr, JBSA Ft. Sam Houston, TX 78234 (miahornberger1@gmail.com). 

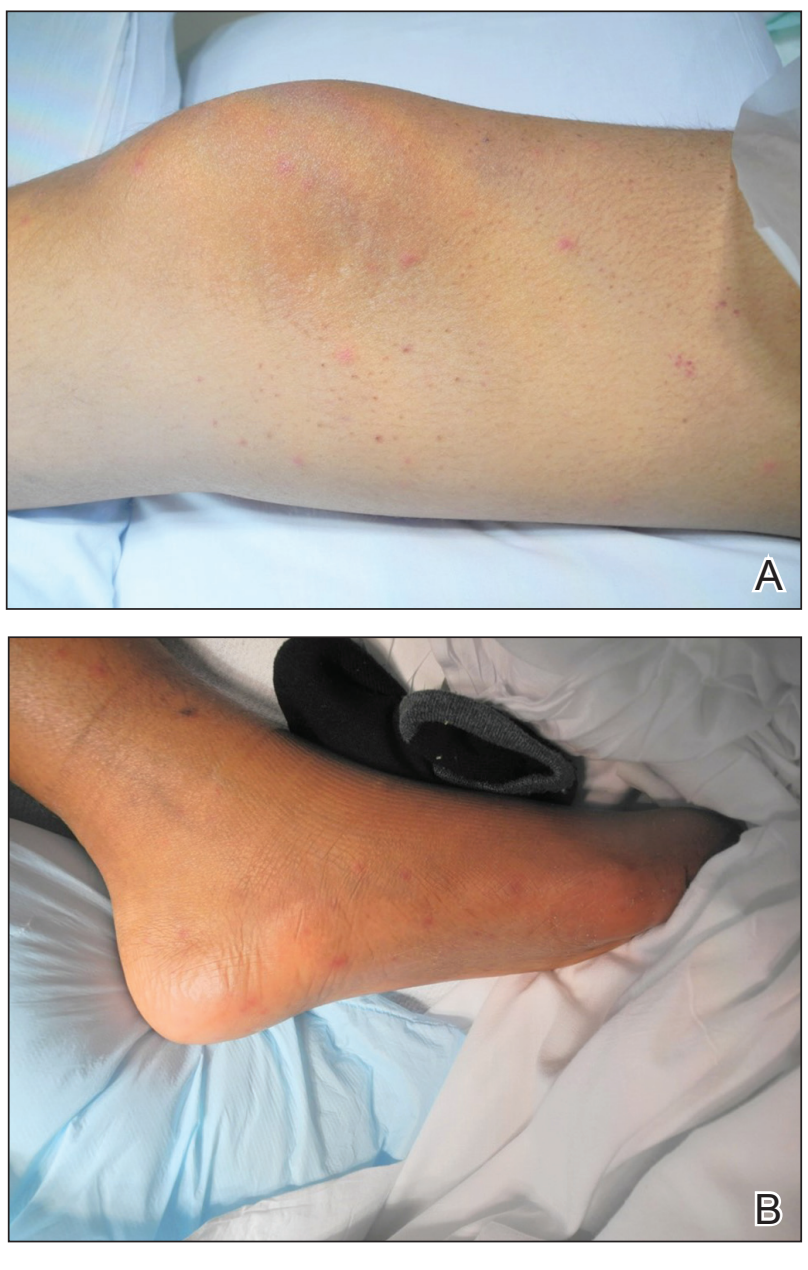

FIGURE 2. A, Multiple discrete pinpoint pink macules on the right leg. B, Faintly erythematous to pink macules on the left ankle and plantar foot.

in the papillary dermis, which was highlighted by periodic acid-Schiff staining with minimal associated inflammation (Figure 3). Blood and tissue cultures persistently grew $C$ tropicalis. The patient was started on intravenous liposomal amphotericin B but died on day 5 of hospitalization after developing endocarditis.

Early and reliable diagnosis of Candida species fungemia is of critical importance to successful treatment, particularly with the emergence of multidrugresistant strains such as Candida auris. ${ }^{2}$ In patients with apparent cutaneous manifestations, a lesional punch biopsy for culture and histopathologic evaluation is recommended in addition to blood culture; however, organisms may or may not be present in large numbers, and they may be difficult to identify on routine hematoxylin and eosin-stained tissue sections. To enhance the likelihood of highlighting the fungus within the sample, the pathologist must be made aware of the presumptive diagnosis of disseminated candidiasis so that special techniques can be utilized, such as periodic acid-Schiff stain.

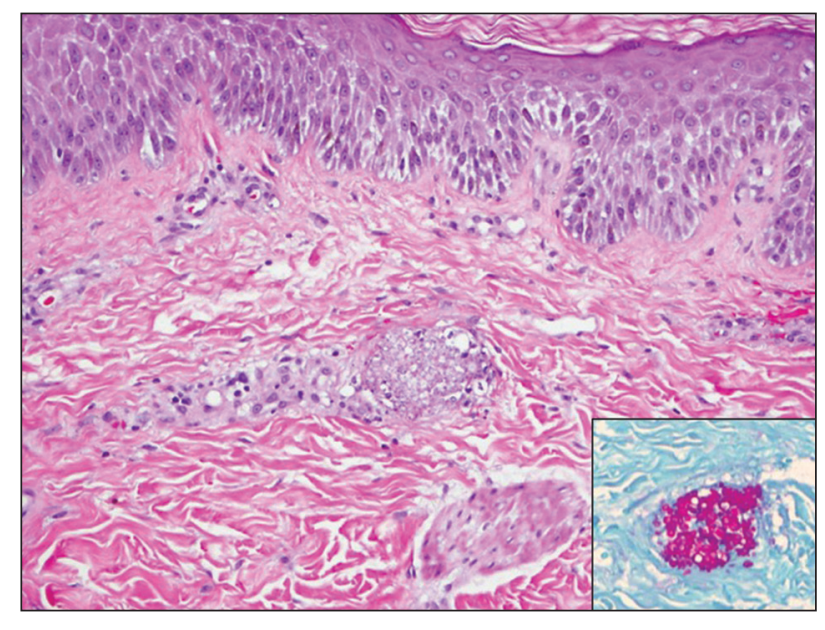

FIGURE 3. A punch biopsy of a lesion on the left dorsal wrist revealed a well-circumscribed, minimally inflammatory collection of yeast (H\&E, original magnification $\times 20$ ), which was highlighted by periodic acid-Schiff stain (inset, original magnification $\times 20$ ).

Although positive blood culture is the gold standard for candidemia diagnosis, only $30 \%$ to $50 \%$ of patients with disseminated candidiasis had positive blood cultures at autopsy. ${ }^{1}$ Another study showed the sensitivity of blood culture for the detection of invasive fungal infection to be as low as $8.3 \% .^{3}$ In cases with positive blood cultures, the median time to positivity is 2 to 3 days, but it can take as long as 8 days, thus limiting its clinical utility in acutely ill patients. ${ }^{4}$ Given the low sensitivity and prolonged time required for culture growth of most fungal organisms, novel assays for rapid, non-culture-based diagnosis of systemic fungal infections hold substantial clinical promise moving forward.

\section{The Technique}

One of the more promising non-culture-based fungal diagnostic methodologies is an antigen assay based on the detection of serum $(1,3)-\beta$-D-glucan (BDG), a major cell wall constituent of most pathogenic fungi. This assay is not specific for Candida species and can be positive for Aspergillosis species, Fusarium species, Coccidioides immitis, Histoplasma capsulatum, and Pneumocystis jirovecii pneumonia, among others; therefore, it functions as a general biomarker for fungi in the bloodstream. ${ }^{4,5}$ $(1,3)$ - $\beta$-D-glucan assay can be useful as an adjunct for blood cultures and punch biopsy, especially when cultures are negative or the results remain outstanding. The results of the BDG assay are available in less than 24 hours at minimal cost, and the test is approved by the US Food and Drug Administration for use as an aid in invasive fungal disease diagnosis. In a metaanalysis of 11 studies, BDG sensitivity was $75 \% .{ }^{4}$ In a study based on autopsy cases from 6 years, BDG specificity was $98.4 \%$ with positive and negative predictive values of $86.7 \%$ and $97.1 \%$, respectively. ${ }^{3}$ Optimal

CONTINUED ON PAGE 36 


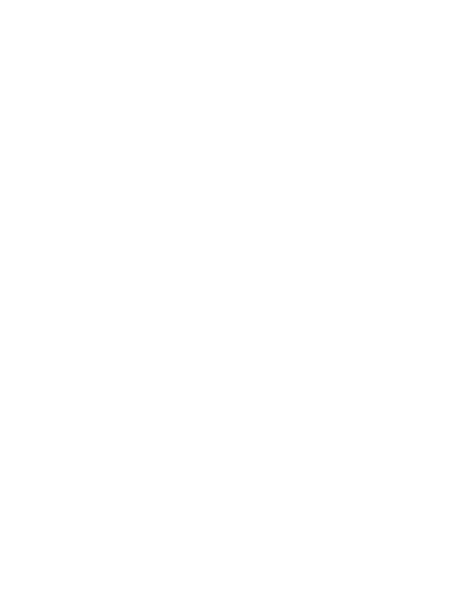

\section{BDG ASSAY}

CONTINUED FROM PAGE 34

results were achieved when 2 consecutive tests were positive. ${ }^{4}$ The serum assay output is based on spectrophotometer readings, which are converted to BDG concentrations (negative, $<60 \mathrm{pg} / \mathrm{mL}$; indeterminate, 60-79 pg/mL; positive $\geq 80 \mathrm{pg} / \mathrm{mL}) .{ }^{5}$ Although we cannot be certain, utilizing the BDG assay in our patient may have led to earlier treatment and a better outcome.

A disadvantage of the BDG assay is the potential for false-positive results, which have been reported in lung transplant recipients with respiratory mold colonization and patients with other systemic bacterial infections. ${ }^{4}$ False-positive results also have been associated with use of ampicillin-clavulanate and piperacillin-tazobactam antibiotics and human blood products, hemodialysis, and severe mucositis, thus reaffirming the importance of judicious interpretation of BDG assay results by the clinician. ${ }^{4,6}$ There also is a potential for false-negative results, as the BDG assay does not detect certain fungal species such as Cryptococcus species and Blastomyces dermatitidis, which produce very low levels of BDG, or zygomycetes (Absidia, Mucor, and Rizopus species), which are not known to produce BDG. ${ }^{6}$

\section{Practice Implications}

In the setting of invasive fungal infections, a high degree of clinical suspicion is paramount due to the often subtle nature of cutaneous manifestations. A positive BDG assay can be used to identify high-risk patients for empiric antifungal therapy, prompting early intervention and improved outcomes in these acutely ill patients. The BDG assay's excellent negative predictive value is useful in ruling out invasive Candida infections and may justify stopping unnecessary empiric antifungal therapy. ${ }^{4}$ For the dermatology hospitalist, incorporation of the BDG assay as a noninvasive screening tool may allow for more rapid initiation of appropriate antifungal therapy while awaiting confirmatory skin biopsy or culture results in disseminated candidemia and other invasive fungal infections.

\section{REFERENCES}

1. Mays SR, Bogle MA, Bodey GP. Cutaneous fungal infections in the oncology patient: recognition and management. Am J Clin Dermatol. 2006;7:31-43.

2. Candida auris. Centers for Disease Control and Prevention website. https://www.cdc.gov/fungal/candida-auris/. Updated May 15, 2020. Accessed July 10, 2020.

3. Obayashi T, Negishi K, Suzuki T, et al. Reappraisal of the serum $(1,3)-\beta$-D-glucan assay for the diagnosis of invasive fungal infectionsa study based on autopsy cases from 6 years. Clin Infect Dis. 2008;46:1864-1870

4. Clancy CJ, Nguyen MH. Finding the "missing 50\%" of invasive candidiasis: how nonculture diagnostics will improve understanding of disease spectrum and transform patient care. Clin Infect Dis. 2013;56:1284-1292.

5. McCarthy MW, Petraitiene R, Walsh TJ. Translational development and application of $(1 \rightarrow 3)-\beta$-d-glucan for diagnosis and therapeutic monitoring of invasive mycoses [published online May 24, 2017]. Int J Mol Sci. doi:10.3390/ijms18061124.

6. Beta-D glucan assay. MiraVista Diagnostics website. https://miravistalabs .com/medical-fungal-infection-testing/antigen-detection/beta-d -glucan-test/. Accessed June 5, 2020. 